\title{
EL AMMI Y CLUSTER EN LA SELECCIÓN DE LOS MEJORES HÍBRIDOS EXPERIMENTALES DE MAÍZ 1
}

\author{
Guillermo Castañón ${ }^{2}$, Rigoberto Zetina ${ }^{3}$, Rafael Arano ${ }^{4}$, Bernardo Raygoza $^{4}$
}

\section{RESUMEN}

El AMMI y Cluster en la selección de los mejores híbridos experimentales de maiz. En un experimento de maíz repetido en cuatro ambientes, se aplicó el análisis de efectos principales aditivos e interacción multiplicativa (AMMI) y el de taxonomía numérica o cluster, para estudiar si la estabilidad del rendimiento de grano (RG) de los híbridos ensayados se asociaba con el agrupamiento de los híbridos hecho por el cluster. Las variables que tuvieron más peso en el agrupamiento fueron el RG, altura de la planta (AP), altura de mazorca ( AM) y mazorca por planta (PRO), principalmente. De lo encontrado en este trabajo se puede establecer que el AMMI es efectivo en la estimación de la estabilidad de los híbridos experimentales evaluados y que junto con el cluster, pueden ser dos herramientas útiles para hacer una buena selección de material experimental con mayores posibilidades de éxito.

\begin{abstract}
The AMMI and Clusters analysis in the selection of the best experimental corn hybrids. Corn hybrids trail was repeated in four different environments, at this was applied the main additive effects and multiplicative interaction analysis (AMMI) and the cluster multivariate analysis or numeric taxonomy, in order to study if the two methods have certain relation to classify for stability and association hybrids performance. Cluster analysis did not show a clear tendency to group to evaluated hybrids for them relatives. According to the results, YG, PH and PRO, were the characteristics more important to joined for evaluated hybrids. The results indicated, that AMMI was effective to determine the stability of experimental hybrids and both (AMMI and Cluster) could be two tools very importants to make a good selection among experimental material.
\end{abstract}

\section{INTRODUCCIÓN}

Entre los grandes retos que han tenido que enfrentar los fitomejoradores de maíz en el último decenio, esta la generación de cultivares que respondan en forma favorable a la aplicación de menor cantidad de insumos como: fertilizantes, plaguicidas y herbicidas, principalemente, pero sin decremento de su nivel productivo. De lograr lo anterior, se estará contribuyendo en forma directa al desarrollo de una agricultura sustentable.

Diferentes métodos se encuentran reportados en la literatura para usarse en la selección de genotipos por su estabilidad, en tanto que para estudiar la asociación o el agrupamiento del germoplasma que se evalúa, se aplica alguno de los métodos desarrollados en la taxonomía numérica.
Una vez demostrada la importancia estadística y genética de la interacción genotipo-ambiente, aparecieron diferentes modelos con los que se pueden estimar los parámetros de estabilidad. Así también, en diferentes trabajos de investigación se ha tratado de comparar la efectividad de una metodología contra otra para seleccionar al mejor material evaluado.

Como ejemplo de lo antes mencionado, se tienen los trabajos desarrollados por Prasad y Shing (1980), quienes compararon los resultados arrojados por las metodologías de Eberhart y Russell (1966), Lewis (1954) y Wricke (1962), en ocho variedades de maíz evaluadas para estabilidad. Los autores reportan, que el rango de los coeficientes de correlación tuvo estrecha similitud entre los dos primeros métodos. El método de Wricke (1962), fue disímil a los otros dos, por usar sólo un parámetro en su clasificación.

1 Presentado en la XLV Reunión del PCCMCA, Guatemala., 1999.

2 Av. José López Portillo \#8, Fracc. Adolfo López Mateos, Campeche, Camp., México. Tel. (981) 2-58-01., e-mail: gcastanonn@yahoo.com.

3 Campo Experimental Papaloapan. Cd. Isla, Ver. México.

4 Centro de Bachillerato Tecnológico Agropecuario \#36, Ignacio de la Llave, Ver., México. 
Por su parte, Chowdhury et al. (1982), al medir la eficiencia de varios métodos de estabilidad en cebada, encontraron similar ubicación o clasificación de los cultivares evaluados entre aquellos métodos que usan en su estimación los parámetros de estabilidad $\beta$ i y $S^{2}$ di.

Referente a estudios de comparación de métodos, en México se tiene el reportado por Castañón y Tosquy (1996), en el que estudiaron la estabilidad del rendimiento de grano de híbridos de maíz con cinco métodos. Los autores reportan que en forma general, las metodologías usadas en su trabajo (Eberhart y Russell, 1966; Wricke, 1962; Shukla, 1972; Tai, 1972 y Eskridge, 1990) mostraron la tendencia de clasificar en el mismo orden a los genotipos evaluados. Y que en los casos en donde si hubo variación de posición en la clasificación, pudo deberse al tipo de parámetros que involucra cada método en la estimación. En otro trabajo, Castañón (1994), comparó en arroz los métodos de Eberhart y Russell (1966) y Wricke (1962), encontró similar clasificación por estabilidad con ambas metodologías.

El AMMI (Análisis de efectos principales aditivos e interacción multiplicativa) es un modelo multivariado que combina en un sólo análisis al de varianza (ANAVA) y al de componentes principales (CP ó PC, en inglés). Las ventajas del AMMI con respecto al de regresión son: El AMMI requiere de menos repeticiones y captura mejor la variación de tratamientos (Gauch, 1993). Además, Gauch y Zobel (1988), señalan que la efectividad del AMMI se incrementa con el tamaño del ensayo y con la reducción del ruido (efectos no estructurales), que es uno de los componentes importantes en los datos que se toman en ensayos conducidos en varias localidades. Adiccionalmente, un mayor número de genotipos pueden ser evaluados sin perder precisión, ni incrementar el costo de los experimentos (Gauch y Furnas, 1991; Crossa et al. 1990).

Quemé et al. (1993), aplicaron el AMMI para seleccionar líneas de maíz evaluadas en ambientes de Centro América, los autores reportaron haber seleccionado las 42 líneas más rendidoras, más estables y con buenas características, así como con cierta resistencia a pudrición de la mazorca.

El AMMI en un ensayo de maíz repetido en varios ambientes contrastantes de Latinoamérica. De sus resultados sobresale el hecho de que el primer componente principal (CP1) retuvo el 28,6\% de la suma de cuadrados correspondientes a la IGA, Córdova (1992). Información similar fue reportada por Crossa et al. (1990) al evaluar dos ensayos internacionales de maíz.

La utilidad del análisis cluster por los fitomejoradores según Shorter et al. (1977) es: a) La identifica- ción de genotipos por su respuesta a los ambientes, éstos pueden usarse como padres y predecirse la respuesta de adaptación de sus progenies. b) Se identifican genotipos con potencial para recomendarse en forma comercial en regiones específicas de producción. c) $\mathrm{Pa}$ ra seleccionar genotipos que se agrupen con cultivares de conocida respuesta, por lo que los nuevos genotipos serán superiores a los primeros. d) El efecto del pedigrí y el origen de los genotipos puede estudiarse a través de los ambientes de evaluación.

Los efectos de la interacción genotipo-ambiente pueden utilizarse para clasificar variedades o genotipos en general, y para clasificar ambientes, Cervantes (1992). De su estudio realizado en 1979 con 25 razas de maíz y las F1 de éstas, reporta que el agrupamiento de los padres mantuvo cierta relación con la altitud de distribución en forma natural de ellos, pero con cierta asociación en relación a su parentesco ancestral.

La selección mediante el cluster, permite al fitomejorador hacer selección de los cultivares superiores, Cornelius et al. (1993). Referente al uso de parámetros de estabilidad y el cluster para seleccionar germoplasma, se tiene el trabajo de Carver et al. (1987), en donde estudiaron la respuesta de híbridos y líneas puras de trigo mediante el uso de la regresión y el análisis cluster.

Por la importancia social y cultural que tiene el maíz en la dieta diaria de la población mexicana, y principalemente de aquella que vive en las áreas rurales. La presente investigación se desarrolló con los objetivos que a continuación se señalan: 1) Seleccionar híbridos experimentales de maíz por su estabilidad en el rendimiento de grano. 2) Observar el patrón de agrupamiento de los híbridos experimentales evaluados en cuatro ambientes contrastantes de producción. 3) Demostrar la posible asociación existente entre la estabilidad y la clasificación dada por el cluster.

\section{MATERIALES Y MÉTODOS}

Campo Experimental Cotaxtla (CECOT): Se localiza en la costa del Golfo de México, a $35 \mathrm{~km}$ del Puerto de Veracruz, de suelos profundos y clima cálido húmedo (Aw1), a los $18^{\circ} 50^{\prime}$ de latitud Norte y 960 10' de longitud Oeste, altitud de $10 \mathrm{msnm}$, temperatura media anual de $24{ }^{\circ} \mathrm{C}$ y precipitación anual de $1300 \mathrm{~mm}$.

Campo Experimental Papaloapan (CEPAP): Se localiza cerca de Cd. Isla, Ver., sus suelos son del tipo cambisol eútrico en su mayoría, de topografía ligeramente ondulada, el clima es Awo. A los $17^{\circ} 45^{\prime}$ de latitud Norte y $95^{\circ} 51^{\prime}$ de longitud Oeste, altitud de 150 
msnm, temperatura entre 24 y $25^{\circ} \mathrm{C}$, con máximas de 41 y mínimas de 12, y precipitación de 1200 mm anuales.

Centro de Bachillerato Tecnológico Agropecuario \# 36 (CBTA 36): Se sitúa a $5 \mathrm{~km}$ de Ignacio de la Llave, Ver., el tipo de suelo es arcillo-arenoso, planos, clima cálido subhúmedo $\left(\mathrm{Aw}_{2}\right)$, a los $18^{\circ} 48^{\prime}$ de latitud Norte y 95० 59' de longitud Oeste, a 7 msnm de altitud, temperatura media de $24,7{ }^{\circ} \mathrm{C}$ y precipitación media anual de $1300 \mathrm{~mm}$.

El ensayo consistió de 38 híbridos experimentales de diferente origen genético y tipo (simples, simples modificados y triples con líneas resistentes a sequía). Se usaron como testigos el H-512 y H-513, que son híbridos comerciales. El material evaluado se agrupó por origen y por tipo, como se muestra en el Cuadro 1.

Cuadro 1. Genealogía de los híbridos experimentales de maíz evaluados en cuatro ambientes del estado de Veracruz, México.1997B y 1998B.

\begin{tabular}{|c|c|}
\hline $\begin{array}{l}\text { Número de } \\
\text { Híbrido }\end{array}$ & Descripción del Cruzamiento \\
\hline 1 y 2 & Simple, línea original y sublíneas de la hembra de H-513. \\
\hline 3 y 4 & Simple, línea original y sublínea del macho de H-513. \\
\hline 5 al 10 & $\begin{array}{l}\text { Triples, líneas sobresalientes en diferentes caracteres } \\
\text { agronómicos. }\end{array}$ \\
\hline 11 y 12 & Cruzas simples modificadas de H-513. \\
\hline 13 al 16 & $\begin{array}{l}\text { Macho modificado de H-513 por líneas sobresalientes } \\
\text { en diferentes caracteres agronómicos. }\end{array}$ \\
\hline 17 al 38 & $\begin{array}{l}\text { Líneas tolerantes a sequía por líneas sobresalientes en } \\
\text { diferentes caracteres agronómicos. }\end{array}$ \\
\hline 39 al 40 & Híbridos comerciales H-512 y H-513. \\
\hline
\end{tabular}

El experimento se estableció como alfa-latice $(0,1)$, la parcela experimental fue de un surco por tratamiento, 3,0 m de longitud, 0,80 $\mathrm{m}$ la distancia entre surcos y 0,20 m la separación entre plantas. A la siembra se depositaron dos semillas por golpe, a los doce días de nacidas las plantas se aclareó a una por mata, para tener 62,5 mil plantas/hectárea. Fueron dos repeticiones por tratamiento en cada ambiente de evaluación. La fecha de siembra en cada ambiente fue la siguiente: El 907-1997 en CEPAP, el 23-07-1997 en CECOT, el 2607-1997 en CBTA \#36, y el 15-07-1998 en CECOT. El manejo agronómico que se dio a los experimentos fue el de una siembra comercial de maíz, se usó la dosis de fertilización 138-46-00 fraccionándola en dos partes, la primera mitad de nitrógeno y todo el fósforo se aplicó a la siembra, y a los 35 dds se proporcionó el resto de nitrógeno. El control de maleza y plagas se realizó en las fechas oportunas y con los productos sugeridos para el cultivo de maíz.
Los caracteres cuantificados fueron: Floración masculina (FM), floración femenina (FF), altura de planta (AP), altura de mazorca (AM), número de mazorcas por planta (PRO), porciento de mazorcas podrídas (MP) y el rendimiento de grano (RG) por parcela se ajusto al $14 \%$ de humedad y por plantas faltantes con la fórmula de Iowa. Hecho lo anterior se expresó en t/ha.

Como el objetivo primordial del programa de maíz del CECOT es encontrar híbridos con amplia adaptación, sólo se consideró el análisis combinado de ambientes, para ello se usó el SAS 6,08 (Statistical Analysis System). Para la corrida del AMMI, se usó el programa MATMODEL escrito en FORTRAN, y para el análisis cluster el NTSYS-PC. En éste último la información se presentó como medias por tratamiento y ambientes de cada variable, éstas se arreglaron en una matriz del orden 40x7, donde las hileras fueron los tratamientos o híbridos y las columnas los siete caracteres.

\section{RESULTADOS Y DISCUSIÓN}

Los resultados del análisis de varianza combinado se presentan en el Cuadro 2; en él puede verse la significación en todos los caracteres medidos en los ambientes de evaluación; es decir, los ambientes de prueba fueron todos diferentes entre sí. El factor híbridos $(\mathrm{H})$ también mostró significación en las características medidas. Mientras que la interacción ambiente $\mathrm{x}$ híbridos $(\mathrm{AxH}$ o IGA), sólo manifestó significancia en FM, FF y RG, que equivale al $43 \%$ de los caracteres medidos. En AP, AM, PRO y MP, se observó un comportamiento paralelo o de no interacción, en la respuesta de los híbridos en estas variables al pasar de uno a otro ambiente de prueba.

El AMMI (Cuadro 2), realizado sólo para la variable RG, identificó como significativos a los componentes principales CP1 y CP2. Los resultados indican que CP1 absorbió el $56 \%$ de la suma de cuadrados de la IGA y el $35 \%$ de los grados de libertad de la misma fuente de variación. Es decir es más importante éste (CP1) que CP2. Por lo que con CP1 es suficiente para representar la interacción de los híbridos con el ambiente.

Cuando se graficó el comportamiento de estabilidad de los materiales evaluados (Figura 1), se encontró que los híbridos 30,15, 12 y 11, se ubicaron entre el germoplasma estable. Además, fueron los que superaron al promedio general de RG. También fueron superiores a H-512, 24 y 7 para el mismo carácter, que fueron los genotipos más estables. En el Cuadro 3, se dan las puntuaciones y el rendimiento promedio de grano de los materiales señalados. En el mismo Cuadro 3, se agregan los híbridos más inestables $(2,3$, y 1$)$, progenitores (excepto el 4) de los híbridos 11 y12, con estos 
Cuadro 2. Cuadrados medios de los caracteres evaluados en los híbridos de maíz. Híbridos 1997B y 1998B. Veracruz, México.

\begin{tabular}{|c|c|c|c|c|c|c|c|c|}
\hline FV & Gl & FM & FF & $\mathbf{A P}$ & $\mathbf{A M}$ & PRO & MP & RG \\
\hline A & 3 & $2182,5 * *$ & $2052,0 * *$ & $10,5 * *$ & $6,0 * *$ & $0,7 * *$ & $1654,7 * *$ & $578,4 * *$ \\
\hline $\mathrm{R} / \mathrm{A}$ & 4 & $5,2 * *$ & $7,8 * *$ & $8,1^{-2} * *$ & $3,4^{-2} * *$ & $0,1 * *$ & $347,6 * *$ & $10,3 * *$ \\
\hline $\mathrm{H}$ & 39 & $23,6 * *$ & $30,3 * *$ & $0,2 * *$ & $0,1 * *$ & $9,9-2 * *$ & $164,7 * *$ & $16,3 * *$ \\
\hline $\mathrm{A} \times \mathrm{H}$ & 117 & $2,5 *$ & $3,4 *$ & $1,2^{-2} \mathrm{~ns}$ & $1,3^{-2} \mathrm{~ns}$ & $2,7^{-2} \mathrm{~ns}$ & $100,1 \mathrm{~ns}$ & $2,0 * *$ \\
\hline CP1 & 41 & & & & & & & $3,3 * *$ \\
\hline CP2 & 39 & & & & & & & $1,5 * *$ \\
\hline RES1 & 37 & & & & & & & 1,2 \\
\hline EE & 156 & 1,7 & 2,5 & $1,4^{-2}$ & $1,2^{-2}$ & $2,4^{-2}$ & 101,0 & 0,8 \\
\hline $\mathrm{X}$ & & 57,5 & 58,2 & 1,8 & 1,0 & 0,9 & 8,1 & 5,7 \\
\hline- & & & & & & & & \\
\hline
\end{tabular}

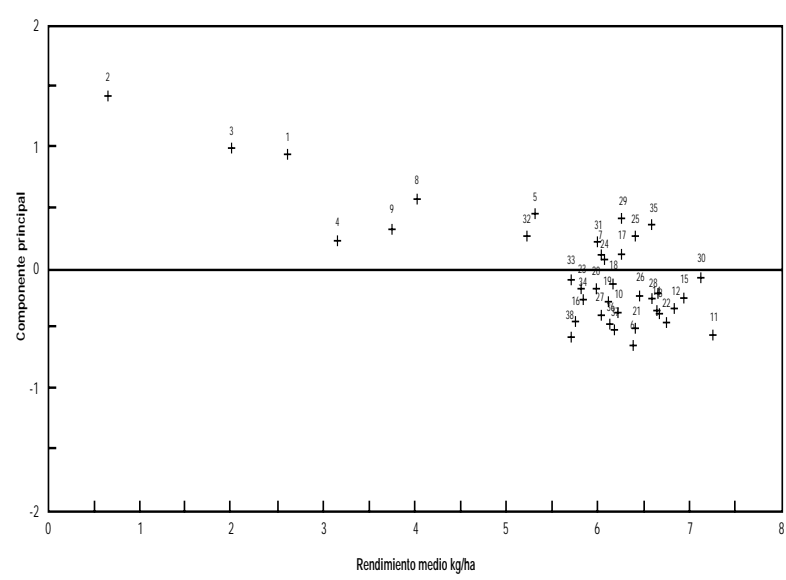

Figura 1. Estabilidad de híbridos de maíz. Veracruz, México. 1997B y 1998B.

resultados se confirma que la estabilidad es heredable y que con uno de los progentiores que sea estable, es suficiente para que el híbrido también lo sea.

El dendograma que muestra la asociación de los híbridos evaluados se presenta en la Figura 2. De acuerdo con ésta, se aprecia que inicialmente los híbridos tendieron a agruparse por la similitud entre los promedios de las variables cuantificadas en el estudio. En cierto modo la mayor importancia para la asociación la mostraron el RG, AP, AM y PRO, esto lo demuestra el grupo formado a 1,4 de distancia por los híbridos 1, 3, 4,9 y 8 , que dieron promedios muy similares de las variables indicadas. Posteriormente la asociación entre los híbridos, puede atribuirse al parentesco existente entre ellos.

Lo anterior se supone, porque en el dendograma se asociaron híbridos que tienen al menos un progenitor en común, por ejemplo, el 19 y 24, 22 y 16, 11 y 12, en éstos últimos la asociación fue a una distancia de
Cuadro 3. Rendimiento de grano de los híbridos estables e inestables de maíz. Híbridos 1997B y 1998B. Veracruz, México.

\begin{tabular}{ccc}
\hline Híbrido & Puntuación & RG (t ha-1) \\
\hline H-512 & 0,006 & 5,87 \\
24 & 0,075 & 6,05 \\
7 & 0,127 & 6,03 \\
$\mathrm{H}-513$ & 0,223 & 6,58 \\
30 & $-0,045$ & 7,20 \\
15 & $-0,224$ & 6,92 \\
12 & $-0,316$ & 6,83 \\
11 & $-0,523$ & 7,25 \\
2 & 1,436 & 0,64 \\
3 & 0,999 & 1,99 \\
1 & 0,957 & 2,60 \\
\hline
\end{tabular}

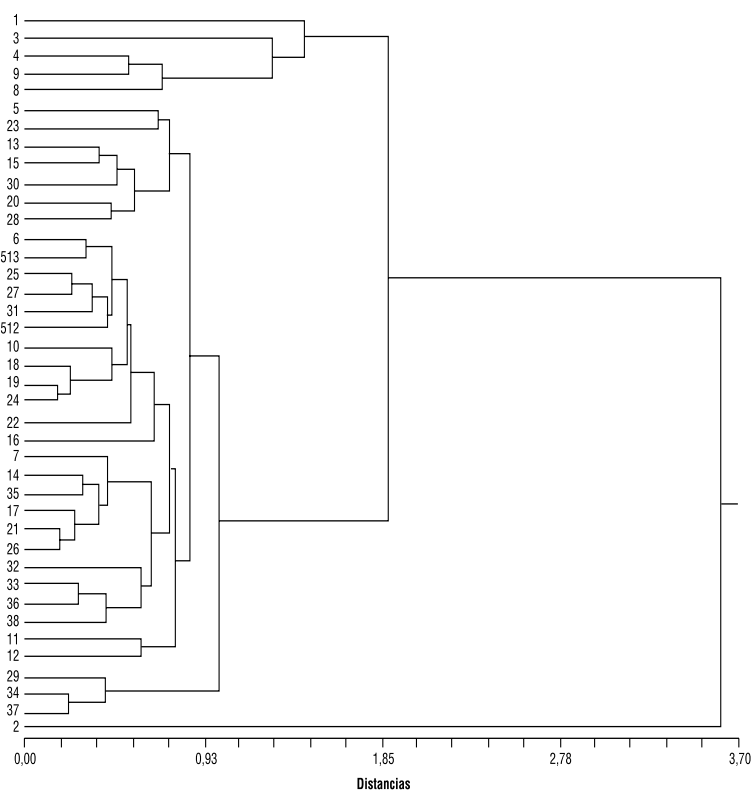

Figura 2. Dendograma de los híbridos experimentales de maíz. 
0,8; ambos híbridos difieren en las sublíneas de cada progenitor de H-513. Otras asociaciones entre híbridos emparentados son el 33 y 36, 34 y 37, que incluyen líneas tolerantes a sequía, puede verse en el dendograma que el germoplasma de sequía fue el que mostró una tendencia más marcada de asociación entre ellos.

Los promedios de los mejores híbridos de todos los caracteres cuantificados se presentan en el Cuadro 4. Una inspección a la información referida, señala que los mejores híbridos superaron ampliamente a los testigos en seis de los siete caracteres. Donde hay poca diferencia es en los híbridos 30, 15 y 12, es para la variable MP, pero el porcentaje de mazorcas podridas no es de consideración, ya que el valor más alto fue de 13,4 $\%$ que correspondió al híbrido 15 , éste apenas duplicó al promedio de MP presentado por H-512.

La superioridad de los mejores híbridos con respecto a los testigos se reafirma de una ligera observación que se haga al Cuadro 5, en donde se puede ver que los testigos presentaron mejores promedios que los peores híbridos. Es decir, entre el material experimental hubo diferencias considerables.

La asociación por el cluster y la estabilidad de los híbridos evaluados en este trabajo, no coincide con lo reportado por Carver et al. (1987), pero si con lo anota-

Cuadro 4. Promedios de los caracteres medidos en los mejores híbridos de maíz. Híbridos 1997B y 1998B. Veracruz, México.

\begin{tabular}{ccccccrc}
\hline Híbrido & FM & FF & AP & AM & PRO & MP & RG \\
\hline 11 & 58,1 & 58,6 & 2,04 & 0,98 & 0,99 & 5,7 & 7,2 \\
30 & 57,6 & 58,1 & 2,03 & 1,06 & 1,03 & 11,5 & 7,2 \\
15 & 57,4 & 57,9 & 2,06 & 1,11 & 0,92 & 13,4 & 6,9 \\
12 & 58,4 & 59,1 & 1,88 & 0,90 & 0,98 & 10,3 & 6,8 \\
22 & 57,2 & 58,2 & 1,88 & 1,09 & 1,04 & 6,5 & 6,7 \\
39 & 57,5 & 58,5 & 1,97 & 1,01 & 0,94 & 7,5 & 6,6 \\
40 & 58,1 & 59,6 & 1,97 & 1,07 & 0,92 & 4,7 & 5,9 \\
\hline
\end{tabular}

Cuadro 5. Promedios de los caracteres medidos en los peores híbridos de maíz. Híbridos 1997B y 1998B. Veracruz, México.

\begin{tabular}{ccccccrr}
\hline Híbrido & FM & FF & AP & AM & PRO & MP & RG \\
\hline 2 & 65,2 & 67,0 & 1,34 & 0,58 & 0,61 & 13,7 & 0,6 \\
3 & 58,4 & 63,7 & 1,57 & 0,74 & 0,67 & 23,9 & 2,0 \\
1 & 62,0 & 63,8 & 1,63 & 0,80 & 0,83 & 9,0 & 2,6 \\
4 & 57,9 & 58,8 & 1,66 & 0,82 & 0,69 & 14,8 & 3,1 \\
9 & 58,1 & 58,5 & 1,63 & 0,87 & 0,77 & 9,7 & 3,7 \\
39 & 57,5 & 58,5 & 1,97 & 1,01 & 0,94 & 7,5 & 6,6 \\
40 & 58,1 & 59,6 & 1,97 & 1,07 & 0,92 & 4,7 & 5,9 \\
\hline
\end{tabular}

do por Ghaderi et al. (1980). Es conveniente hacer notar que los autores citados usaron para su estudio el análisis cluster y la estimación de la estabilidad con el modelo de Eberhart y Russell y como variable de respuesta sólo al rendimiento de grano. Salmerón et al. (1996), usaron el AMMI para agrupar cultivares de avena, los autores mencionan que la agrupación dada por el AMMI se basó en la resistencia de los genotipos a las enfermeddes y al periodo de madurez.

Al hacer la comparación entre ambientes, se puede apreciar (Cuadro 6) que los promedios de los caracteres AM, PRO, MP y RG, fueron mejores en el ambiente del CECOT. En tanto que el CBTA 36, mostró promedios intermedios en todas las variables y CEPAP fue el peor ambiente de prueba. La explicación a lo anterior es que los híbridos repondieron a lo pobre del ambiente, el CEPAP es suelo ácido.

Cuadro 6. Promedios por ambiente en los caracteres evaluados en los híbridos de maíz. Híbridos, 1997B y 1998B. Veracruz, México.

\begin{tabular}{ccrrr}
\hline Cáracter & CECOT & CBTA 36 & CEPAP & CECOT \\
\hline FM & $55,0 \mathrm{c}$ & $54,1 \mathrm{~d}$ & $65,3 \mathrm{a}$ & $55,6 \mathrm{~b}$ \\
FF & $55,0 \mathrm{c}$ & $56,4 \mathrm{~b}$ & $65,8 \mathrm{a}$ & $55,8 \mathrm{~b}$ \\
AP & $2,0 \mathrm{ab}$ & $1,9 \mathrm{~b}$ & $1,3 \mathrm{c}$ & $2,0 \mathrm{a}$ \\
AM & $1,1 \mathrm{a}$ & $1,1 \mathrm{a}$ & $0,6 \mathrm{c}$ & $1,0 \mathrm{~b}$ \\
PRO & $1,0 \mathrm{a}$ & $0,8 \mathrm{~b}$ & $0,8 \mathrm{~b}$ & $1,0 \mathrm{a}$ \\
MP & $2,4 \mathrm{a}$ & $8,4 \mathrm{~b}$ & $13,5 \mathrm{a}$ & $8,0 \mathrm{~b}$ \\
RG & $8,2 \mathrm{a}$ & $4,4 \mathrm{c}$ & $2,6 \mathrm{~d}$ & $7,7 \mathrm{~b}$ \\
\hline
\end{tabular}

Promedios con la misma letra en hileras son estadísticamente iguales $(\mu=0,05)$.

La superioridad de los híbridos más estables con respecto a la media general de rendimiento de grano fue imperceptible. En tanto que los híbridos más rendidores mostraron aceptable estabilidad y superaron a los testigos en rendimiento de grano, así como en el resto de los caracteres evaluados. Los peores híbridos o de menor producción, fueron los más inestables y superados ampliamente por los testigos. El componente principal uno (CP1), indicó la presencia de considerable interacción entre los híbridos y los ambientes de evaluación. Al parecer, el cluster agrupó primero a los híbridos por la importancia de los caracteres (RG, AP, AM, PRO, etc.). Posteriormente, por el parentesco del material y la similitud entre los promedios de los caracteres evaluados.

Los híbridos simples modificados (11 y 12) del H513 , superaron a éste en todas las cracterísticas evaluadas. No obstante de que no hubo una tendencia clara en los resultados arrojados por el AMMI y el Cluster, se percibe que la combinación de ambos métodos es efectiva para seleccionar al material experimental superior. 
Los híbridos que se formaron con líneas resistentes a sequía, mostraron buen comportamiento y alto potencial de rendimiento.

\section{LITERATURA CITADA}

CARVER, B. F.; SMITH, E. L.; ENGLAND Jr. H. O. 1987. Regression and cluster analysis of environmental responses of hybrid and pureline winter wheat cultivars. Crop Sci. 27:659-664.

CASTAÑ́́N, N. G.; TOSQUY, V. O. H. 1996. Análisis de la interacción Genotipo-Ambiente en híbridos tropicales de maíz (Zea mays L.) con diferentes métodos. Rev. Fitot. Mex. 19:141-150.

CASTAÑÓN, N. G. 1994. Estudio de la estabilidad de líneas avanzadas y variedades de arroz usando dos metodologías. Agron. Mesoam. 5:118-125.

CERVANTES, S. T. 1992. La interacción genotipo-ambiente en la clasificación de regiones agrícolas. Memorias, Simposio Interacción Genotipo-Ambiente en Genotecnia Vegetal. Guadalajara, Jal., México. pp. 117-125.

CÓRDOVA, H. 1992. Resultados diferenciales para rendimiento de híbridos de maíz evaluados en ambientes contrastantes de Latinoamérica. Agron. Mesoam 3:1-8.

CORNELIUS, P. L.; VAN SANFORD, D. A.; SEYEDSADR, M. 1993. Clustering cultivars into groups without rankchange interactions. Corp Sci. 33:1193-1200

CROSSA, J.; GAUCH JR. H. G.; ZOBEL, R. W. 1990. Additive main effects and multiplicative interaction analysis of two international maize cultivar trails. Crop. Sci. 30(3):493-500.
CHOWDHURY, R. K; SINGH, V. P. ; SINGH, R. K. 1982. Efficiency of various stability models for ranking Barley Genotypes. Cereal Research Communications. 10(2):95-101.

GHADERI, A.; EVERSON, E. H.; CRESS, C. E. 1980. Classification of environments and genotypes in wheat. Crop Sci. 20:707-710

GAUCH, JR. H. G. 1993. Prediction, parsimony and noise. American Sci. 81:468-478.

GAUCH, JR. H. G.; ZOBEL, R. W. 1988. Predictive and posdictive sucess of statistical of yield trials. Theor. Appl. Genet. 76:1-10.

GAUCH, JR. H. G.; FURNAS, R. E. 1991. Statistical analysis of yield trails with MATMODEL. Agron. J. 83:916-920.

PRASAD, S K.; SINGH, T. P. 1980. A comparison of different methods for determining the stability of maize varieties. Indian J. Agric. Sci. 50(10):731-733.

QUEMÉ, J. L.; PÉREZ, C.; FUENTES, M.; LARIOS, L.; CASTELlANOS, S. 1993. Selección de líneas de maíz (Zea mays L.) de grano blanco para factores adversos bióticos y abióticos en seis localidades de Centro América. XXXIX Reunión Anual del PCCMCA. ICTA, Cd. Guatemala, Guatemala.

SALMERÓN, Z. J. J.; CABAÑAS, C. B.; CHÁVEZ, CH. J.; VALENZUELA, V. M. 1996. Agrupación de ambientes de temporal y genotipos de avena con el modelo AMMI. Rev. Fitotec. Mex. 19(2):151-162.

SHORTER, R.; BYTH, D. E. ; MUNTGOMERY, V. E. 1977. Genotype $\mathrm{x}$ environment interactions and environmental adaptation. II. Assessment of environmental contributions. Aust. J. Agric. Res. 28:233-235. 